\title{
GNSS precise point positioning in regional reference frames using real-time broadcast corrections
}

\author{
Lennard Huisman, ${ }^{1, *}$ Peter J. G. Teunissen ${ }^{1,2}$ and \\ Congwei $\mathrm{Hu}^{1}$ \\ ${ }^{1}$ GNSS Research Centre, Curtin University of \\ Technology, Australia \\ ${ }^{2}$ DEOS, Delft University of Technology, The Netherlands
}

\begin{abstract}
As a result of the Real-Time IGS Pilot Project (RT-IGS) and the EUREF Real-Time Analysis Project (EUREF-RT), real-time GNSS satellite orbit and clock corrections to broadcast ephemeris, the so-called real-time broadcast corrections (BC), have become available for the application in precise point positioning (PPP). The realtime BCs are currently available in global as well as in regional reference frames. In this contribution the PPP usage and performances of these global and regional BCs are analysed for the Australian continent. We identify the limitations of the current regional $\mathrm{BCs}$, which results in scale-induced biases, and show how they can be improved. We also present an alternative, more user friendly, approach for regional BCs. It provides PPP users with simple means to correct for the biases of the regional approach.
\end{abstract}

Keywords. Global navigation satellite systems, precise point positioning, global and regional reference frames, global and regional broadcast corrections.

\section{Introduction}

Precise point positioning (PPP), first described in [26], is a global navigation satellite system (GNSS) positioning method that processes undifferenced pseudorange and carrier phase measurements from a stand-alone GNSS receiver to compute positions with a high, decimeter or centimeter, accuracy everywhere on the globe. By using satellite orbits and clocks, as well as other corrections (for Earth rotation, tides and ocean loading, phase wind-up, etc.), the GNSS antenna position along with other parameters, like atmospheric delays, can be estimated $[12,16]$. In recent years, services have been developed which allow high accuracy ephemeris data to be made available in real-time to users

Corresponding author: Lennard Huisman, GNSS Research Centre, Curtin University of Technology, Australia.

E-mail: p.teunissen@curtin.edu.au.

Received: October 3, 2011. Accepted: December 31, 2011.
$[4,6,11,22]$. Such availability has created, and will continue to create, a wide range of PPP applications $[3,10]$.

An important example of such services are the realtime GNSS satellite orbit and clock corrections to broadcast ephemeris, the so-called real-time broadcast corrections (BCs). They have become available since early 2008 as a result of the Real-Time IGS Pilot Project (RT-IGS) and the EUREF Real-Time Analysis Project (EUREF-RT) [21]. The main aim of these BCs is to provide users with precise corrections to broadcast orbits and clocks for the application in PPP. Three years later, mid 2011, the available BCs not only give users more precise orbit and clock information, but next to a global reference frame (GRF) they are now also available in regional reference frames (RRF), such as the European ETRF2000, the North-American NAD83 or the Australian GDA94 [4,20], see Figure 1.

In this contribution we will analyse the usage of these regional broadcast corrections (RBCs) and compare their performance with the usage of the more traditional global broadcast corrections (GBCs). Since our analyses are done for PPP in the Australian region, the geodetic datum GDA94 is taken as the RRF.

This contribution is organised as follows. In Section 2 we describe the usages of global and regional BCs for precise point positioning. Since we consider PPP in the Australian region, we also describe the GRF-to-RRF transformation for the Australian geodetic datum GDA94. In Section 3 we analyse and compare real-time PPP using global and regional $\mathrm{BCs}$. We identify the limitations of the current RBC-approach and show how it can be improved. In Section 4, we present a new, more userfriendly, approach for regional BCs. It eliminates the time-varying receiversatellite dependent position biases of the current RBCapproach and it provides the PPP user with simple means to correct for the remaining small biases.

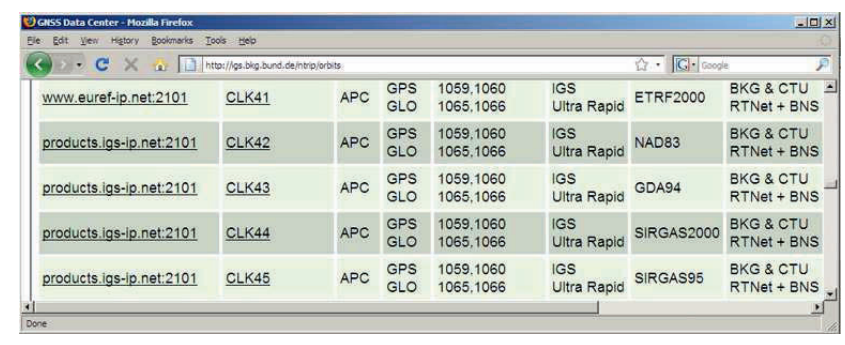

Figure 1. An example list of available real-time BCs in regional reference frames [4]. 


\section{Precise point positioning in regional reference frames}

In this section we describe the two approaches that are currently available for realising real-time PPP in regional reference frames.

\subsection{The global and regional broadcast corrections}

Satellite positions are usually given in a global reference frame (GRF) such as WGS84 (GPS broadcast ephemeris), PZ90.2 (GLONASS broadcast ephemeris) or IGS05 (IGS orbits). Rather than having one's position in a GRF, a PPP user is often interested in obtaining his final positions in a local or regional reference frame (RRF), like, for instance, ETRF2000 (Europe) [5], NAD83 (North-America) [7], SIRGAS2000 (South-America) [9] or GDA94 (Australia) [8]. To obtain the user's position in an RRF, a frametransformation has to be applied from the GRF, in which the satellite positions are given, to the RRF. Currently, a user can follow two different approaches for determining one's RRF position coordinates:

(a) the global broadcast correction (GBC) approach, or

(b) the regional broadcast correction (RBC) approach.

The GBC-approach is the traditional one. Tools have been available for the RBC-approach for many years (e.g. [13]). Through the RT-IGS and EUREF-RT projects the RBCapproach has become available in real-time [20]. These two approaches are schematically depicted in Figure 2.

Traditionally the transformation from a GRF to an RRF is applied at the user level (see Figure 2 (a)). In this GBCapproach, precise orbits and clocks (box 1 of Figure 2(a)) are used to generate global broadcast corrections (box 3) to the broadcast ephemeris (box 2). The PPP-algorithm (box 4) uses these GBCs and the broadcast ephemeris together with the GNSS Observations (box 5) to compute a precise receiver antenna position in the GRF (box 6). A GRF-to-RRF coordinate transformation (box 7) is then finally applied to obtain the receiver antenna position in the required RRF (box 8).

In scope of the RT-IGS and EUREF-RT projects it has been proposed to make $\mathrm{BCs}$ available that directly give the satellite positions in an RRF $[4,21]$. The basic idea behind these regional BCs is the thought that when processing stand-alone GNSS data the reference frame in which the user's position is obtained is defined by the realisation of the reference system in which the satellite positions are given. The benefit of a regional $\mathrm{BC}$ product is that a user does not have to transform his position anymore, but will get his position directly in the desired RRF via the RBCapproach (Figure 2(b)). The main difference between the two approaches is thus that with the RBC-approach, the GRF-to-RRF transformation is already performed by the Server that computes the $\mathrm{BCs}$ rather then by the individual User.

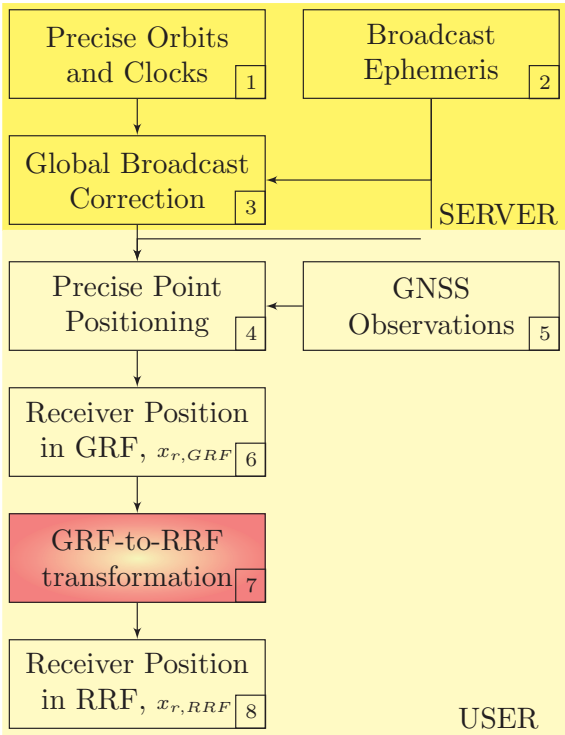

(a)

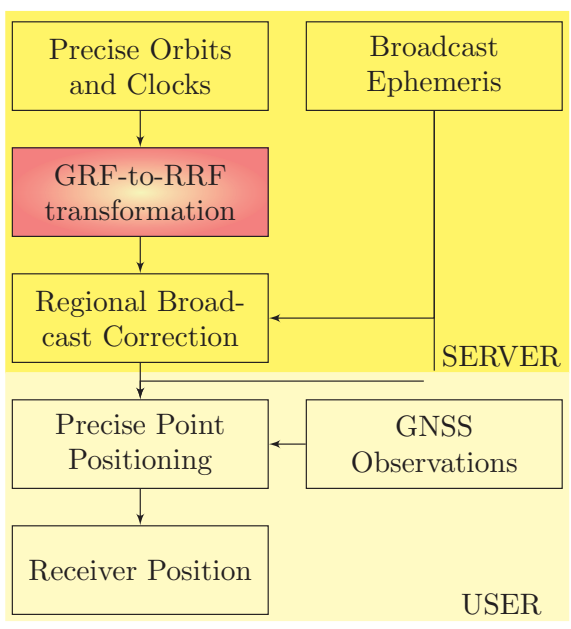

(b)

Figure 2. Flow diagram for obtaining a PPP position in an RRF. (a) The GBC-approach: USER-side GRF-to-RRF transformation. (b) The RBC-approach: SERVER-side GRF-to-RRF transformation.

\subsection{The GRF-to-RRF transformation}

A coordinate transformation is needed from the GRF to the RRF, so as to obtain RRF position coordinates. This GRFto-RRF transformation at an epoch $t$ is performed by a 7parameter similarity transformation:

$$
x_{\mathrm{RRF}}(t)=d(t)+s(t) R(t) x_{\mathrm{GRF}}(t),
$$


where

$$
\begin{aligned}
d(t) & =\left[\begin{array}{l}
d_{x}(t) \\
d_{y}(t) \\
d_{z}(t)
\end{array}\right], \\
s(t) & =(1+\Delta s(t)), \\
R(t) & =\left[\begin{array}{ccc}
1 & -r_{z}(t) & r_{y}(t) \\
r_{z}(t) & 1 & -r_{x}(t) \\
-r_{y}(t) & r_{x}(t) & 1
\end{array}\right],
\end{aligned}
$$

with

$x_{\mathrm{GRF}}$ coordinate vector in the global frame (GRF),

$x_{\mathrm{RRF}}$ coordinate vector in the regional frame (RRF),

$d \quad$ vector with translation parameters $d_{x}, d_{y}, d_{z}$,

$s \quad$ scale factor between GRF and RRF,

$\Delta s \quad$ increment of $s$ to 1 ,

$R \quad$ matrix with differential rotation angles $r_{x}, r_{y}, r_{z}$.

The transformation parameters are often considered to be dependent on time, in which case their time dependency needs to be known as well. Usually it is sufficient to only consider their linear time dependency. In that case the transformation is referred to as a 14-parameter transformation. The 14 parameters consists then of the 7 similarity transformation parameters, plus their 7 time-rates of change, all given at a certain reference epoch $t_{0}$. These 14 parameters can then be used to compute the 7 similarity transformation parameters for any epoch $t$ as

$$
\begin{aligned}
d(t) & =d\left(t_{0}\right)+\left(t-t_{0}\right) \dot{d}\left(t_{0}\right), \\
R(t) & =R\left(t_{0}\right)+\left(t-t_{0}\right) \dot{R}\left(t_{0}\right), \\
\Delta s(t) & =\Delta s\left(t_{0}\right)+\left(t-t_{0}\right) \Delta \dot{s}\left(t_{0}\right),
\end{aligned}
$$

with

$\dot{d} \quad$ rate of change of the translation vector,

$\dot{R} \quad$ rate of change of the rotation matrix,

$\dot{\Delta S} \quad$ rate of change of the scale factor.

\subsection{The Australian datum and GDA94-RBCs}

As our analysis in subsequent sections will be given for the Australian GDA94, we now give the defining parameters for this geodetic datum.

The Australian GDA94 datum is a realisation of the International Terrestrial Reference Frame (ITRF), specifically ITRF1992 at the reference epoch of 1 January 1994 [8]. In the generation of the GDA94-RBCs, the transformation is based on the ITRF2005 [2] to GDA94 parameters [4]. This is a 14-parameter transformation and it is performed by using equation (1). The transformation parameters at the epoch of observations are computed with equation (2),

\begin{tabular}{ll}
\hline$t_{0}[\mathrm{yr}]$ & 1994.0 \\
\hline$d_{x}\left(t_{0}\right)[\mathrm{mm}]$ & -79.73 \\
$d_{y}\left(t_{0}\right)[\mathrm{mm}]$ & -6.86 \\
$d_{z}\left(t_{0}\right)[\mathrm{mm}]$ & 38.03 \\
$r_{x}\left(t_{0}\right)[\mathrm{mas}]$ & 0.0351 \\
$r_{y}\left(t_{0}\right)[\mathrm{mas}]$ & -2.1211 \\
$r_{z}\left(t_{0}\right)[\mathrm{mas}]$ & -2.1411 \\
$\Delta s\left(t_{0}\right)[\mathrm{ppb}]$ & 6.636 \\
$\dot{d}_{x}\left(t_{0}\right)[\mathrm{mm} / \mathrm{yr}]$ & 2.25 \\
$\dot{d}_{y}\left(t_{0}\right)[\mathrm{mm} / \mathrm{yr}]$ & -0.62 \\
$\dot{d}_{z}\left(t_{0}\right)[\mathrm{mm} / \mathrm{yr}]$ & -0.56 \\
$\dot{r}_{x}\left(t_{0}\right)[\mathrm{mas} / \mathrm{yr}]$ & -1.4707 \\
$\dot{r}_{y}\left(t_{0}\right)[\mathrm{mas} / \mathrm{yr}]$ & -1.1443 \\
$\dot{r}_{z}\left(t_{0}\right)[\mathrm{mas} / \mathrm{yr}]$ & -1.1701 \\
$\Delta \dot{s}\left(t_{0}\right)[\mathrm{ppb} / \mathrm{yr}]$ & 0.294 \\
\hline
\end{tabular}

Table 1. Transformation parameters from ITRF2005 to GDA94 (adopted from [8]).

from the parameters given in Table 1. Note that the rotation matrix of equation (1) is given according to Chapter 4 of the IERS conventions [18], whereas a different definition for the rotation matrix is used in [8], which results in a change of the sign in the rotation parameters and their rates.

Also note that the IGS satellite orbits, on which all current $\mathrm{BCs}$ are based, are given in the IGS realisation of the ITRF, e.g., in IGS05 from November 2006, or since April 2011, in IGS08. For the transformation from IGS05/IGS08 to the RRF the ITRF2005/2008 to RRF transformation parameters can be applied, since the transformation parameters between IGS05/08 and ITRF2005/2008 are zeros by default [1].

\section{Precise point positioning in GDA94}

In this section we compare the performances of the GBCand RBC-approaches for real-time PPP in the GDA94.

\subsection{Real-time PPP with global BCs}

The PPP method, using IGS products and the necessary correction models, is described in [12]. For the handling of the ionospheric delays, different options are available. In [12] and [15], the unknown ionospheric delays are eliminated from the observation equations by using appropriate linear combinations of either the dual-frequency or the single-frequency phase and code observations. In [16], [14] and [24], on the other hand, external information from Global Ionospheric Maps (GIM) [19] are used to 


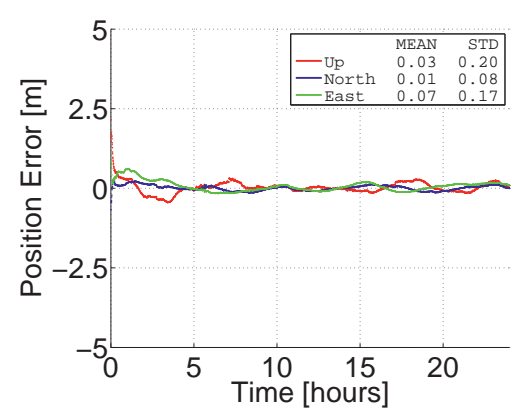

(a) Dual-frequency ionosphere-free

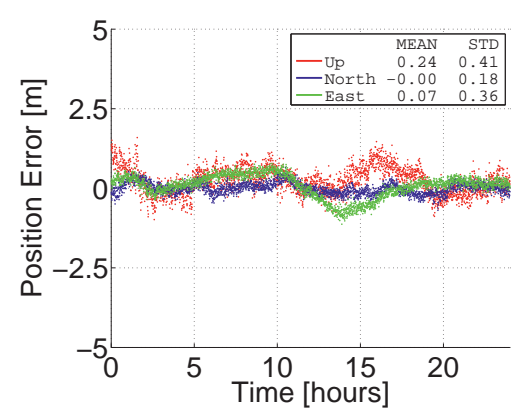

(b) Single-frequency ionosphere-free

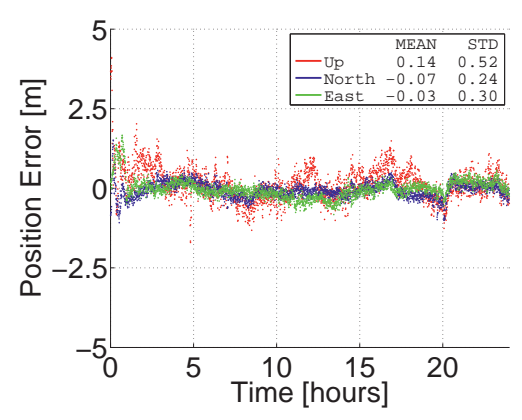

(c) Single-frequency with GIM correction

Figure 3. Typical North-East-Up PPP performance (with BKG clocks): ionosphere-free and GIM-corrected.

a-priori correct the single-frequency observations for the ionospheric delays.

In our real-time PPP-software we have implemented all three ionospheric variants, i.e., the two ionospherefree variants (dual-frequency and single-frequency) and the single-frequency, GIM corrected variant. Their typical realtime GDA94-GBC PPP performances are shown in Figure 3 for BKG clock products [25]. Both the dual- and singlefrequency ionosphere-free variants have a convergence of several hours, whereas the single-frequency variant with GIM corrections shows little convergence time. The precision after convergence for the dual-frequency case is at sub-decimetre level both horizontally and vertically, while single-frequency achieves less than half-meter precision for the vertical and a few decimetre in the horizontal component.

\subsection{Real-time PPP with regional BCs}

Now we present our real-time PPP results using the GDA94-RBCs as obtained from the RT-IGS NTRIP caster. The analysis has been performed for four stations on the Australian continent (Darwin (DARM), Townsville (TOW2), Hobart (HOB2) and Perth (CUT0)). Figure 4 shows the locations of these stations. Stations DARM, TOW2 and HOB2 are part of the Australian Regional GPS Network [23] and operated by Geoscience Australia, CUT0 is operated by Curtin University. For the GBC data processing the BKG CLK11 product has been used, which gives BC referring to IGS05, the RBC results are based on the BKG CLK43 product referring to GDA94. The results shown are for 20 March 2011 with a 1 second sampling rate. For all stations the analysis leads to the same conclusions. We therefore only show the dual-frequency ionosphere-free results for the Australian CORS station, CUT0, located at Curtin University, Perth. The receiver at CUT0 is a Trimble NetR9 connected to a Trimble GNSS Choke-Ring antenna with a Unavco SCIS radome (TRM59800.00 SCIS).

Since the RBC-approach aims at providing a PPP user position in the RRF, the positioning results of the two

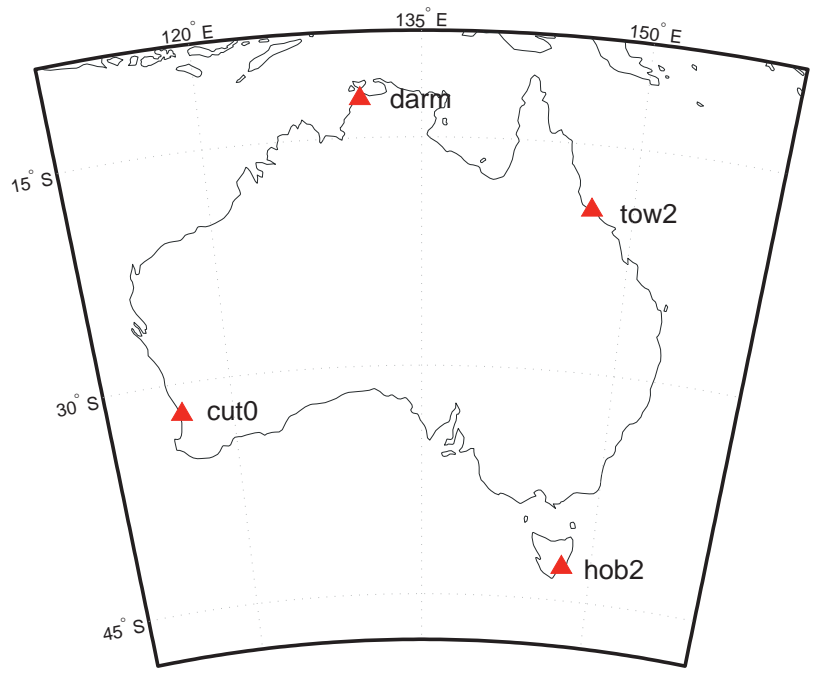

Figure 4. CORS stations on the Australian continent used for the analysis of real-time PPP using BCs.

approaches, GBC and RBC, are compared at the coordinate level in the RRF. All settings of our software were identical in the PPP processing of the GBCs an the RBCs; only the BCs input differed. Figure 5 shows the position differences in GDA94 for both approaches. All position components show systematic errors that are slowly changing over time.

In the horizontal component the biases are up to $10 \mathrm{~mm}$ and for the up component the error is between 55 and $80 \mathrm{~mm}$. This shows that the RBC-approach of making satellite positions directly available in an $\operatorname{RRF}[4,21]$ does not give the PPP-user's position directly in that same RRF. Hence, the originally thought benefit of the regional BCs, namely that a PPP-user would not have to transform his position anymore when using the RBC-approach, is not correct.

The explanation for this identified pitfall follows when one considers the PPP-algorithm itself. A closer look at the effect of the transformation on the positioning algorithm reveals, that although the rotation and translation do 


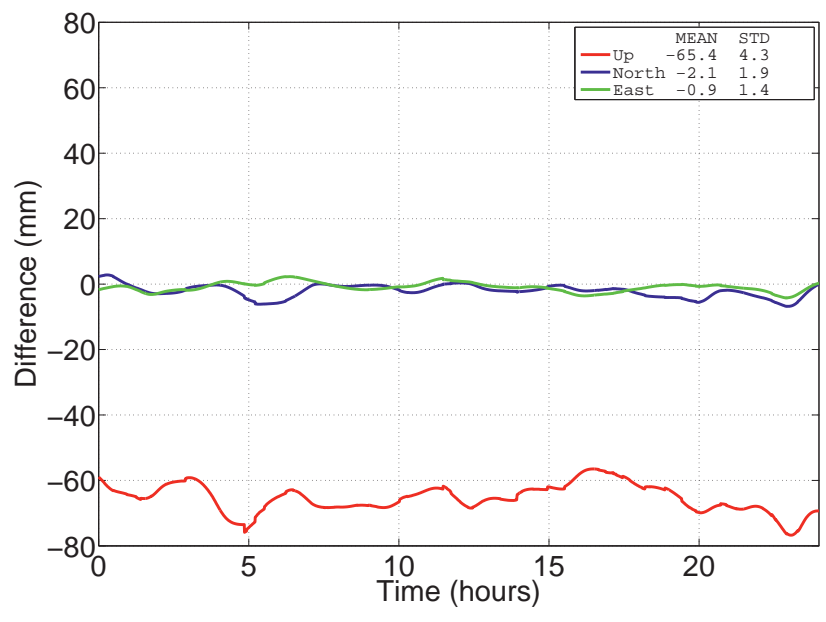

Figure 5. North-East-Up coordinate differences between using the GBC-approach and RBC-approach.

not affect the PPP algorithm, the scaling of course does (see equations (3) and (4) below). Hence, one can only expect identical results between the two approaches if the GRF and the RRF have the same scale. Since this is not the case between ITRF2005 and GDA94, scale-induced, timevarying receiver-satellite geometry dependent biases will be present in the RBC-approach, as is shown in Figure 5. Note that such biases will also remain present in case the $\mathrm{RBC}$-approach is applied to relative positioning, although the effect will then get smaller for shorter baselines, as is shown in [8].

\subsection{Scale-corrected PPP ranges for bias-free RBCs}

In order to correct for the above described scale-induced biases, an appropriate modification of the phase and code observation equations in the PPP algorithm is needed. Let $\rho_{r, \mathrm{GRF}}^{s}$ and $\rho_{r, \mathrm{RRF}}^{s}$ denote the receiver-satellite ranges in the GRF and RRF, respectively. Then these ranges and their increments are related as

$$
\begin{aligned}
\rho_{r, \mathrm{GRF}}^{s} & =\left\|x_{\mathrm{GRF}}^{s}-x_{r, \mathrm{GRF}}\right\| \\
& =\frac{1}{s}\left\|x_{\mathrm{RRF}}^{s}-x_{r, \mathrm{RRF}}\right\|=\frac{1}{s} \rho_{r, \mathrm{RRF}}^{s}
\end{aligned}
$$

and

$$
\Delta \rho_{r, \mathrm{GRF}}^{s}=-\frac{1}{s}\left(u_{r, \mathrm{RRF}}\right)^{T} \Delta x_{r, \mathrm{RRF}}
$$

with $x_{\mathrm{GRF}}^{s}, x_{r, \mathrm{GRF}}$ and $x_{\mathrm{RRF}}^{s}, x_{r, \mathrm{RRF}}$, the satellite and antenna position vectors in GRF and RRF, respectively, and $u_{r, \mathrm{RRF}}$ being the unit direction vector from receiver antenna to satellite and $\Delta x_{r, \mathrm{RRF}}$ the increment vector of the antenna position, both expressed in the RRF.

Equations (3) and (4) show how the PPP observation equations need to be modified so as to obtain bias-free RRF positioning results with the RBC-approach. Figure 6 (a)

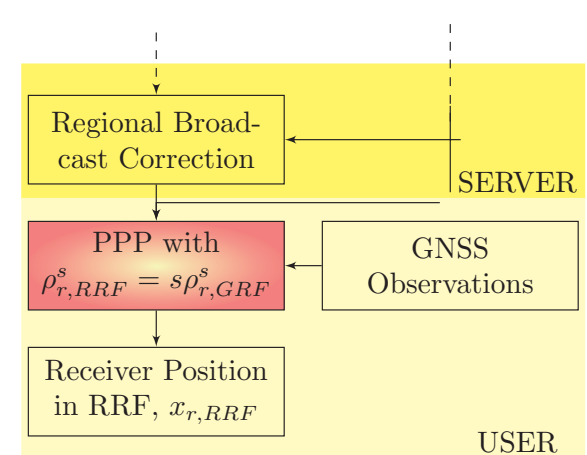

(a)

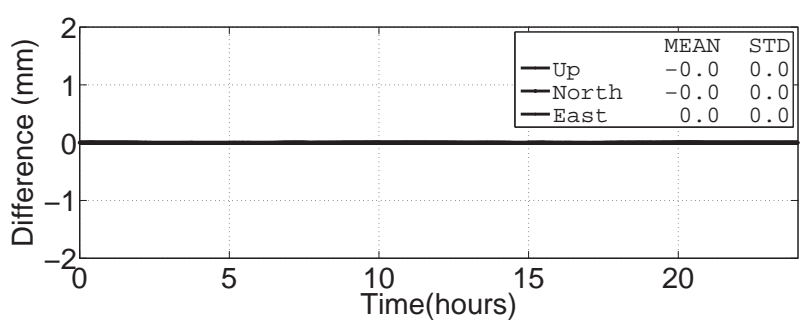

(b)

Figure 6. (a) Location in flow diagram of modified RBC-approach where scale-factor has to be applied. (b) N-E-U coordinate differences between using the GBC-approach and the modified RBC-approach, i.e., after the user has included the scale-correction in his PPP-Algorithm (compare with Figure 5).

depicts this change to the flow diagram and Figure 6 (b) shows the results of processing the data using this modified approach. The systematic differences between the solutions of the two approaches disappear once the scale-factor is properly included in the PPP algorithm.

\section{A new approach to regional broadcasting corrections}

As shown above, the difference in scale between a GRF and an RRF results in receiver-satellite geometry dependent biases when using the current RBC-approach for PPP. In the previous section it was shown that one will have to modify one's PPP-algorithm so as to eliminate these scale-induced biases of the RBC-approach. In this section we propose an alternative, more user-friendly RBC-approach.

\subsection{Real-time PPP with unscaled RBCs}

Instead of applying the complete GRF-to-RRF transformation to the orbital corrections, we propose to apply only the rotation and translation, but not the scale. By excluding the scale, one can avoid the rather difficult to predict, time-varying receiver-satellite geometry dependent biases 


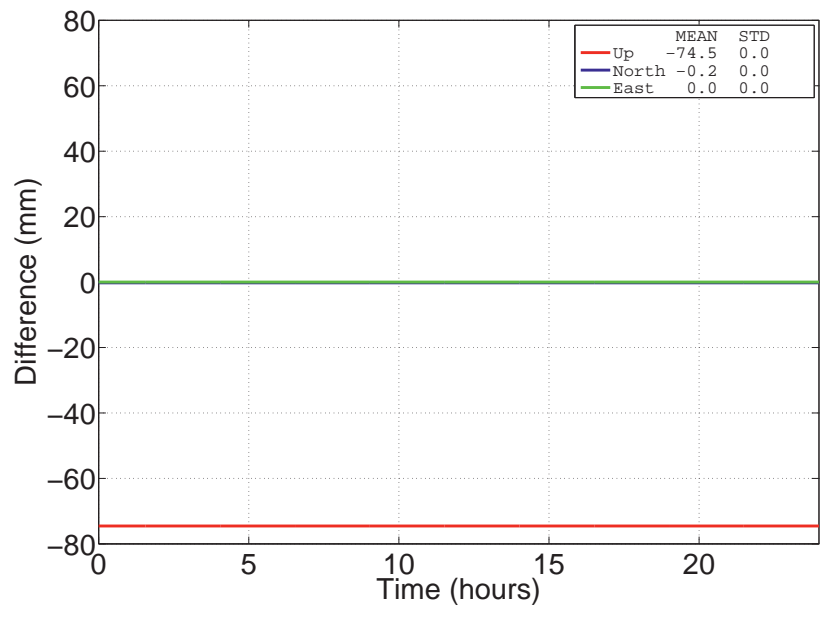

(a)

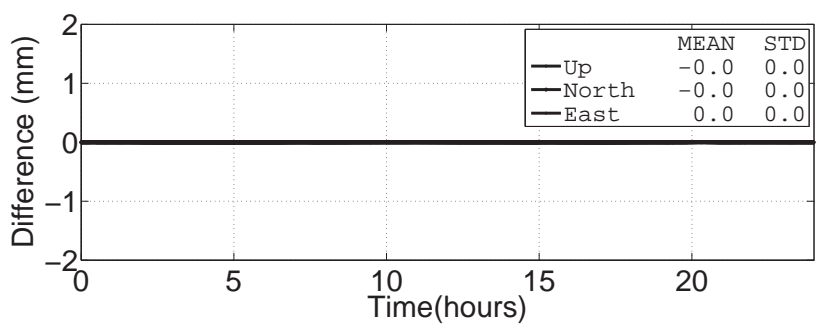

(b)

Figure 7. (a) N-E-U coordinate differences between using the GBC-approach and the unscaled RBC-approach. (b) N-E-U coordinate biases vanish after unscaled RBC based PPP position vector is scaled to GDA94's RRF (compare with Figure 5).

that are present in the current RBC-approach. By using RBCs that still have the scale of the GRF, one achieves that the remaining biases become easy to predict and easy to correct for. Hence, with this approach, a user will not have to modify his PPP-algorithms. Instead, it then suffices to apply a simple a-posteriori scalar correction. In fact, for many applications, this a-posteriori correction would not even be needed.

Figure 7 (a) shows the results of applying the unscaled RBC-approach for station CUT0 in GDA94. When this result is compared to that of Figure 5, we note that the timevarying, receiver-satellite geometry dependent biases of the $\mathrm{RBC}$-approach have now been replaced by biases that are independent of this time-changing geometry. These remaining biases are solely due to the scale difference between the GRF and the RRF. Hence, by applying this scale (in our case the ITRF2005-to-GDA94 scale) to the computed position vector, one obtains the PPP solution in GDA94 and the difference with the solution of the GBC-approach has disappeared, see Figure 7 (b). Hence, the resulting position is then identical to the 'classical' GBC-approach for which the complete GRF-to-RRF transformation is applied a posteriori at the user side.

\subsection{The effect of scale on GDA94 positions}

Although the biases that are shown in Figure 7 (a) are independent of the time-varying receiver-satellite geometry, they are still dependent on the location of the receiver antenna. They therefore vary from location to location. This location dependent effect can be quantified by analysing the effect scale has on position.

We now analyse for the Australian continent the scaling effect on GDA94 positions. We start with the relation between Cartesian and ellipsoidal coordinates,

$$
\left[\begin{array}{l}
x \\
y \\
z
\end{array}\right]=\left[\begin{array}{c}
(N+h) \cos \phi \cos \lambda \\
(N+h) \cos \phi \sin \lambda \\
\left(N\left(1-e^{2}\right)+h\right) \sin \phi
\end{array}\right]
$$

with the East-West and North-South radii of curvature given as

$$
N=\frac{a}{\left(1-e^{2} \sin ^{2} \phi\right)^{1 / 2}}, \quad M=\frac{a\left(1-e^{2}\right)}{\left(1-e^{2} \sin ^{2} \phi\right)^{3 / 2}}
$$

and the eccentricity computed from the major axis $a$ and minor axis $b$ of the ellipsoid as

$$
e=\sqrt{\frac{a^{2}-b^{2}}{b^{2}}}
$$

Linearising and then inverting (5) gives

$$
\left[\begin{array}{c}
\Delta h \\
(M+h) \Delta \phi \\
N \cos \phi \Delta \lambda
\end{array}\right]=R(\phi, \lambda)\left[\begin{array}{c}
\Delta x \\
\Delta y \\
\Delta z
\end{array}\right]
$$

with the orthogonal matrix

$$
R(\phi, \lambda)=\left[\begin{array}{ccc}
\cos \phi \cos \lambda & \cos \phi \sin \lambda & \sin \phi \\
-\sin \phi \cos \lambda & -\sin \phi \sin \lambda & \cos \phi \\
-\sin \lambda & \cos \lambda & 0
\end{array}\right]
$$


When the coordinates of a point are scaled with $\Delta s(s=$ $1+\Delta s$ ), we have for the change in its Cartesian coordinates

$$
\left[\begin{array}{c}
\Delta x \\
\Delta y \\
\Delta z
\end{array}\right]=\Delta s\left[\begin{array}{c}
(N+h) \cos \phi \cos \lambda \\
(N+h) \cos \phi \sin \lambda \\
\left(N\left(1-e^{2}\right)+h\right) \sin \phi
\end{array}\right] .
$$

Substitution of (10) into (8) gives

$$
\left[\begin{array}{c}
\Delta h \\
(M+h) \Delta \phi \\
N \cos \phi \Delta \lambda
\end{array}\right]=\Delta s\left[\begin{array}{c}
N\left(1-e^{2} \sin ^{2} \phi\right)+h \\
-N e^{2} \sin \phi \cos \phi \\
0
\end{array}\right] .
$$

This result shows that position vector scaling only affects the North-South and height components, but not - due to the rotational symmetry of the ellipsoid - the East-West component. Equation (11) also shows how the scaling effect can be computed for any scale at any epoch $t$ if the scale is time dependent.

Figure 8 shows, for the whole of Australia, the effect of the ITRF2005-GDA94 scale on the North-South and height components for epoch 1st March 2010. Based on the Australian Digital Terrain Model (Figure 8 (a)), the formulas $(M+h) \Delta \phi=-N e^{2} \sin \phi \cos \phi \Delta s$ and $\Delta h=$ $\left(N\left(1-e^{2} \sin ^{2} \phi\right)+h\right) \Delta s$ of equation (11) have been used to compute the necessary North-South and height corrections. They are shown in Figure 8 (b) and (c). Hence, these are the corrections that need to be applied to the PPP solution when no a posteriori scaling is applied to the solution of the unscaled RBC-approach.

It can be seen that the effect of the scale factor is constant over Australia at the level of $0.2 \mathrm{~mm}$ for each component. The height correction, for instance, is not very sensitive to height differences, because of the very small scale. Since $\Delta s \approx 7 \times 10^{-9}$, a change in height of $h=10 \mathrm{~km}$, only changes the height correction by $\Delta h \approx 0.07 \mathrm{~mm}$. The correction itself is smaller than $0.3 \mathrm{~mm}$ in North-South direction and approximately $74 \mathrm{~mm}$ in the height direction. Hence, just like the scale factor $\Delta s$ is location independent, one can also consider the coordinate-pair $\Delta h$ and $(M+h) \Delta \phi$ to a large extent location independent (the third component is identically zero: $N \cos \phi \Delta \lambda \equiv 0$ ). Note that equation (11) is generally valid and that it can therefore be used to infer the scale-induced effect for any RRF. The smaller the scale-difference, as is the case with for instance ETRF2000 or SIRGAS2000, the smaller the scale-induced effect of equation (11).

\subsection{PPP with unscaled RBCs and a height correction}

Even more straightforward than applying the scale correction to the user's position vector would be the application of only a height correction or even not a correction at all. Since it was shown that for the Australian continent the unscaled RBC's height bias is sufficiently constant and that

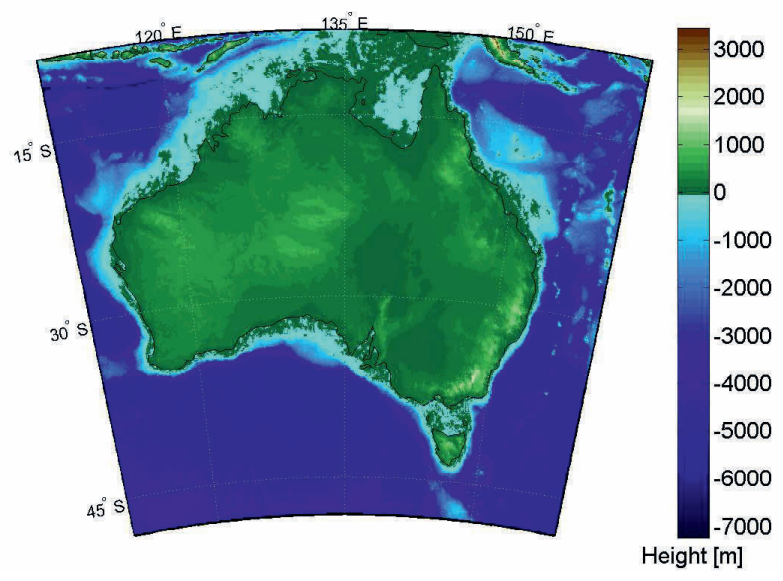

(a)

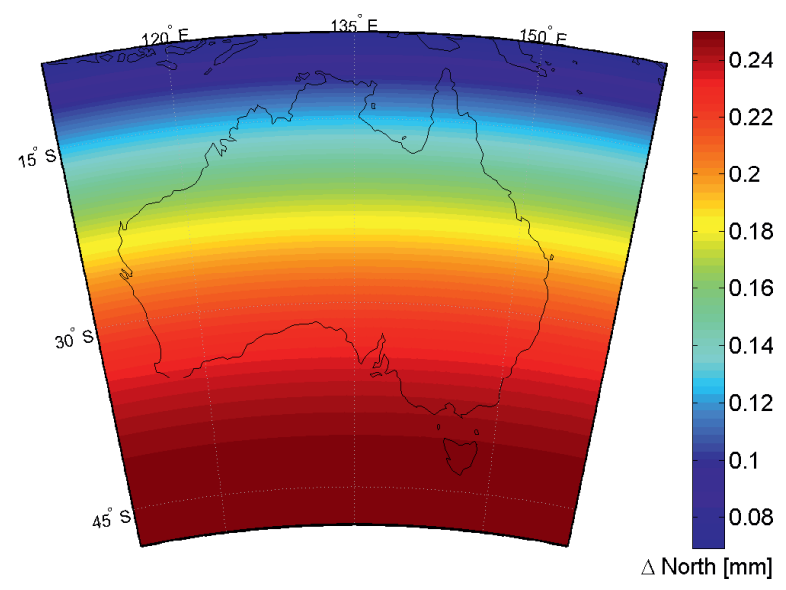

(b)

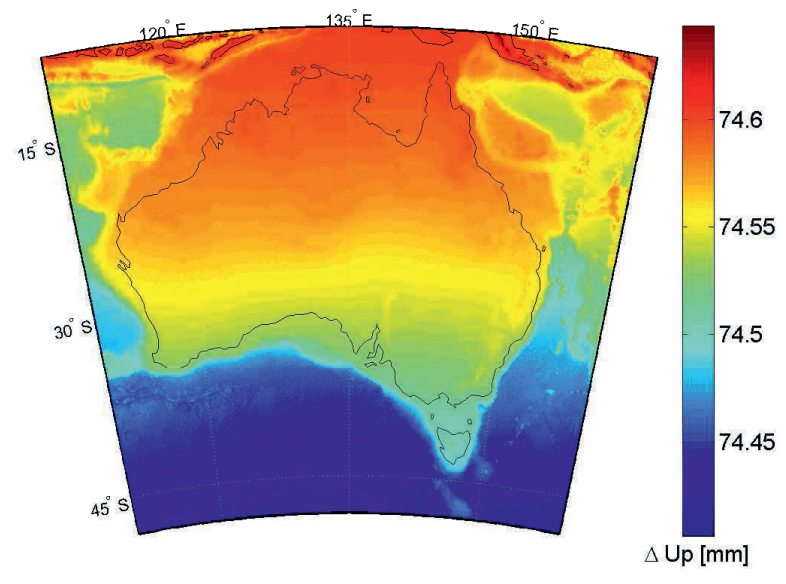

(c)

Figure 8. (a) Australian digital terrain model, derived from global DTM2006.0 [17]; Australian, 1st March 2010, North-South (b) and height (c) correction when using the unscaled RBC-approach without a posteriori scale correction. 


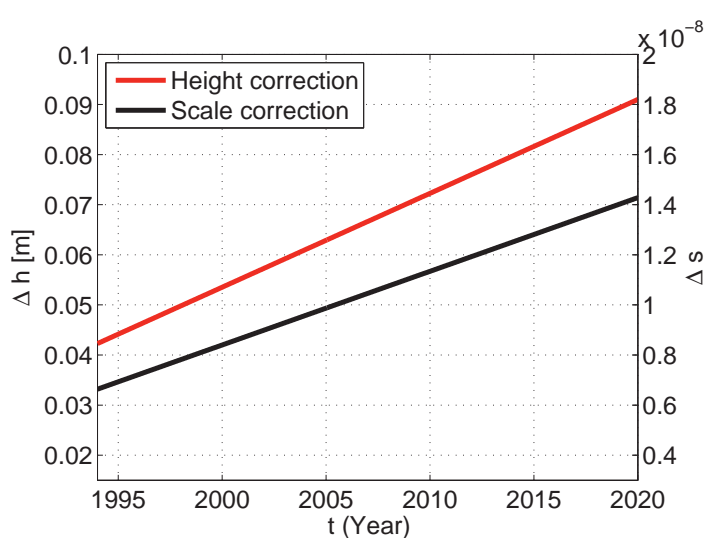

Figure 9. GDA94 height correction $\Delta h(t)$ and scale correction $\Delta s(t)$ as function of time.

its North-South bias is smaller than $0.3 \mathrm{~mm}$, the usage of a single constant height correction to the user's position would result, for all practical purposes, given the current PPP accuracies, in a bias-free user position. No correction at all would even be needed for those applications for which only the horizontal positioning is of interest.

The necessary correction (scale-factor or height correction) has to be supplied to the user though, for instance by inclusion in the BC-stream. In the case of GDA94 the correction is time-dependent since the scale-factor is timedependent. The necessary time-dependent scale-factor or height-correction can be computed for any epoch $t$ from the values of Table 1 using equations (2) and (11). Figure 9 shows the values of these corrections as function of time. The scale-effect between ITRF2005 and GDA94 causes a modest increase in the height bias of $1.87 \mathrm{~mm} / \mathrm{yr}$ (or $9.4 \mathrm{~mm}$ per 5 year) for which the unscaled RBC PPP-solution can be exactly corrected for. In a similar way it can be shown that also the change over time of the North-South bias is small. For the time period 1995-2020, for instance, it stays within the $0.1-0.3 \mathrm{~mm}$ range.

\section{Summary and conclusions}

Real-time precise point positioning (PPP) requires real-time precise corrections to broadcast orbits and clocks. These real-time broadcast corrections (BCs) are currently not only available in a global reference frame (GRF), the global BCs (GBCs), but also as regional BCs (RBCs) in regional reference frames (RRF), such as ETRF2000, NAD83 and GDA94.

In this contribution, we analysed the PPP-usage of the RBCs for positioning in the Australian geodetic datum GDA94. GPS data from stations around Australia were collected and analysed, thus enabling a PPP user coordinatelevel comparison between the $\mathrm{RBC}$-approach and the traditional GBC-approach.

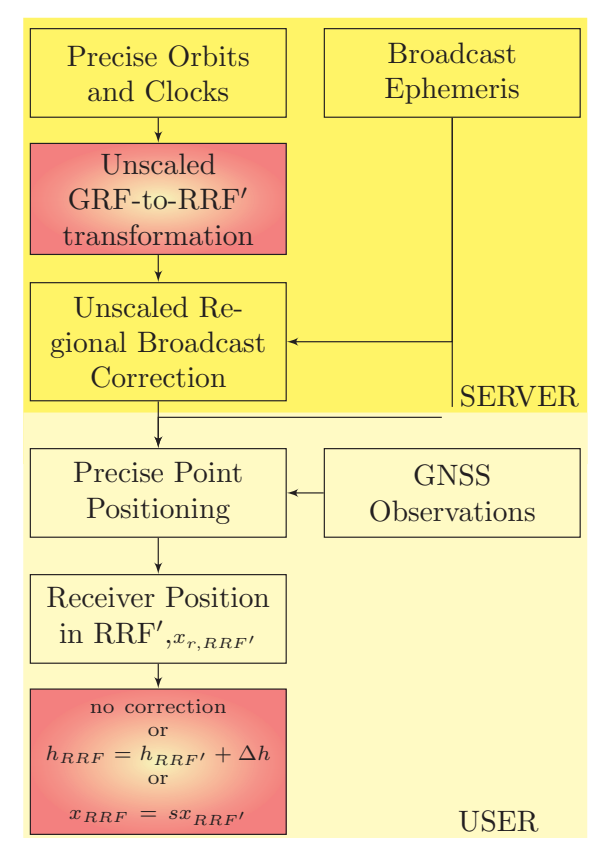

Figure 10. Flow diagram of the proposed unscaled RBCapproach for GDA94, with the three user-options: (1) no a posteriori user correction; (2) only a height correction; or (3) a vectorial scale correction.

Our analysis revealed limitations of the current RBCapproach. In our comparison of the two approaches, position differences of up to $80 \mathrm{~mm}$ showed up for all stations. It was proven that these time-varying, receiver-satellite geometry dependent position biases are caused by scale differences between the GRF and the RRF, in our case between the ITRF and GDA94. Hence, for an RBC-approach to provide results that are identical to the traditional GBCapproach, it is not enough to only provide the $\mathrm{BCs}$ in a regional reference frame as is currently done.

As it was shown, one way to eliminate the position biases from the current RBC-approach is to modify one's PPPalgorithms, thereby equipping the phase and code observation equations with the necessary scale factor. This approach, although rigourous, may not be very attractive from a user's point of view. We therefore proposed in this contribution an alternative, more userfriendly approach.

Instead of applying the complete GRF-to-RRF transformation to the broadcast correction, we propose to only apply the rotation and translation, but not the scale. With this unscaled RBC-approach, users will not have to modify their PPP algorithms. Instead, it then suffices to apply a simple a posteriori scale correction. In fact, as it was shown numerically, for many applications one can even do with only a height correction or not a correction at all. The flow diagram of the proposed unscaled RBC-approach is summarised in Figure 10. 


\section{Acknowledgments}

The second author is the recipient of an Australian Research Council (ARC) Federation Fellowship (project number FF0883188). Part of this work was done in the framework of the project 'New Carrier-Phase Processing Strategies for Next Generation GNSS Positioning' of the Cooperative Research Centre for Spatial Information (CRC-SI2). The real-time PPP software used to process data for this contribution is a modified version of the Single-Frequency PPP software developed at Delft University of Technology. The Australian digital terrain model was extracted from the global DTM2006.0 for this work by Dr. Christian Hirt. All this support is gratefully acknowledged.

\section{References}

[1] Z. Altamimi, Personal communication, 2011.

[2] Z. Altamimi, X. Collilieux, J. Legrand, B. Garayt and C. Boucher, ITRF2005: A new release of the international terrestrial reference frame based on time series of station positions and earth orientation parameters, Journal of Geophysical Research 112 (2007), B9.

[3] S. Bisnath and Y. Gao, Current state of precise point positioning and future prospects and limitations, in: Observing Our Changing Earth, International Association of Geodesy Symposia 133, Springer (2008), 615-623.

[4] BKG, Real-time GNSS satellite orbit and clock corrections from IGS and EUREF sources, http://igs.bkg.bund. de/ntrip/orbits, 2010.

[5] C. Boucher and Z. Altamimi, The EUREF terrestrial reference system and its first realizations, in: EUREF Meeting, Bern (1992), 4-6.

[6] CODE, Center for Orbit Determination in Europe, University of Berne, www.aiub.unibe.ch/ionosphere.html, 2006.

[7] M. R. Craymer, The evolution of NAD83 in Canada, Geomatica 60 (2006), 151-164.

[8] J. Dawson and A. Woods, ITRF to GDA94 coordinate transformations, Journal of Applied Geodesy 4 (2010), 189-199.

[9] H. Drewes, K. Kaniuth, C. Völksen, S. M. Alves Costa and L. P. Souto Fortes, Results of the SIRGAS campaign 2000 and coordinates variations with respect to the 1995 South American geocentric reference frame, A Window on the Future of Geodesy (2005), 32-37.

[10] P. Heroux, Y. Gao, J. Kouba, F. Lahaye, Y. Mireault, P. Collins, K. Macleod, P. Tetreault and K. Chen, Products and applications for precise point positioning - moving towards real-time, Proc. ION GNSS ITM (2004), 1832-1843.

[11] J. Kouba, A guide to using international GPS service (IGS) products, Publication of the IGS Central Bureau, http: // igscb.jpl.nasa.gov/igscb/resource/pubs, 2003.
[12] J. Kouba and P. Heroux, Precise point positioning using IGS orbit and clock products, GPS Solutions 5 (2001), 12-28.

[13] J. Kouba, The GPS toolbox ITRF transformations, GPS Solutions 5 (2002), 88-90.

[14] A. Q. Le and C. Tiberius, Single-frequency precise point positioning with optimal filtering, GPS Solutions 11 (2007), 61-69.

[15] O. Montenbruck, Kinematic GPS positioning of LEO satellites using ionosphere-free single frequency measurements, Aerospace Science and Technology 7 (2003), 396-405.

[16] O. Øvstedal, Absolute positioning with single-frequency GPS receivers, GPS Solutions 5 (2002), 33-44.

[17] N. K. Pavlis, J. K. Factor and S. A. Holmes, Terrain-related gravimetric quantities computed for the next EGM, in: Proceedings of the 1st International Symposium of the International Gravity Field Service, Harita Dergisi, Istanbul (2006), 318-323.

[18] G. Petit, B. Luzum et al. (eds.), IERS Conventions (2010), IERS Technical Note No. 36, Verlag des Bundesamts für Kartographie und Geodäsie, Frankfurt am Main, 2010.

[19] S. Schaer, Mapping and predicting the earth's ionosphere using the global positioning system, Ph.D. thesis, AIUB, University of Berne, Berne, 1999.

[20] W. Söhne, EPN special project "Real-Time Analysis", status report, www. eurefiag.net/TWG/EUREF\% 20TWG\%20minutes/53-Gavle2010/07-b_euref2010_ realtime_soehne.pdf, 2010.

[21] W. Söhne, A. Sturze and G. Weber, Enhancement of EPN real-time data streams, draft, Www. epncb.oma.be/_organisation/projects/RT_ analysis/enhancement_realtime.pdf, 2008.

[22] P. Tetreault, J. Kouba, P. Heroux and P. Legree, CSRS-PPP: An internet service for GPS user access to the canadian spatial reference frame, Geomatica 59 (2005), 17-28.

[23] R. J. Twilley and P. W. Digney, The Australian regional GPS network, Physics and Chemistry of the Earth, Part A: Solid Earth and Geodesy 26 (2001), 629-635.

[24] R. van Bree and C. Tiberius, Real-time single-frequency precise point positioning: accuracy assessment, GPS Solutions (2011), 1-8.

[25] G. Weber, L. Mervart, Z. Lukes, C. Rocken and J. Dousa, Real-time clock and orbit corrections for improved point positioning via NTRIP, in: Proceedings of the 20th International Technical Meeting of the Satellite Division of The Institute of Navigation (ION GNSS 2007), Fort Worth, TX (2007), 1992-1998.

[26] J. Zumberge, M. Heflin, D. Jefferson, M. Watkins and F. Webb, Precise point positioning for the efficient and robust analysis of GPS data from large networks, Journal of Geophysical Research 102 (1997), 5005-5017. 